\title{
Regulación chilena para las actividades turísticas en la Antártica ${ }^{1}$
}

\section{Chilean Legal regulation for Tourism in Antarctica}

\section{Carolina Flores Barros}

flores.barrosc@gmail.com

Abogada, Universidad de Chile. Ayudante del Departamento de Derecho Internacional de la Facultad de Derecho de la Universidad de Chile.

Resumen: Mientras las cifras del turismo antártico aumentan y las actividades que la componen se diversifican, los riesgos para el medioambiente antártico son cada vez mayores. Ante ello, el Sistema del Tratado Antártico ha establecido un marco regulatorio, a través de la Evaluación del Impacto sobre el Medio Ambiente. El presente artículo describe la regulación de esta materia en el Sistema del Tratado Antártico y cómo Chile la ha implementado a nivel político y jurídico.

Palabras claves: Antártica - Evaluación de Impacto Ambiental - Turismo - Sistema del Tratado Antártico - Protocolo del Medio Ambiente - IAATO

\begin{abstract}
As tourism rates in Antarctica increases, and its activities becomes more diverse, the environmental risks rises. The Antarctic Treaty System provides a legal framework, through Environmental Impact Assessment. This paper describes how this topic has been regulates within the Antarctic Treaty System and how Chile has implemented it in its policies and law.
\end{abstract}

Keywords: Antarctica - Environmental Impact Assessment - Tourism - Antarctic Treaty System - Environmental Protocol - IAATO

\section{Introducción}

Al caracterizar la actividad turística en la Antártica, se puede observar que sus cifras aumentan rápidamente. No solo en cuanto al número de turistas que arriban al continente blanco, cuyo número fue 1.000 turistas en la temporada 1957-1958, ascendiendo a 27.000 turistas en la

1 Ponencia presentada en las Jornadas Chilenas de Derecho Antártico 2019, Facultad de Derecho, Universidad de Chile, 28 de marzo del 2019.

Número de página no utilizable para citar 
Regulación Chilena para las actividades turísticas en la Antártica Carolina Flores Barros

temporada 2003-2004 y 45.808 personas en la temporada 2017-2018 (Bastmeijer y Roura, 2004: 763), sino que también las actividades que se realizan se han ido diversificando. Hoy en día la experiencia turística contempla ofertas que incluyen nuevas experiencias, que pueden ir desde maratones, escalada de montañas, paseos en kayak, buceo, snowboard, hasta recolección de meteoritos, entre otras.

La mayoría de los turistas llegan al sexto continente a través de embarcaciones, predominando aquellos cruceros con un máximo de 500 pasajeros. También existe turismo aéreo que llega hacia las Islas Shetland del Sur, donde embarcan en cruceros.

En el contexto anterior, existe cierta preocupación en torno a esta actividad, que conlleva riesgos, tales como la alteración del medioambiente y sus organismos, a través de la introducción de especies no nativas, contaminación acústica (causada por la circulación del transporte aéreo, marítimo y terrestre), cambios físicos en el medioambiente, alteración de la fauna (sobre todo en sus temporadas de reproducción), derrame de petróleo, liberación de gases desde las naves, entre otros. Los riesgos anteriores se ven aumentados por el hecho de que las actividades sean concentradas en el tiempo y el espacio: se realizan en la temporada estival, principalmente en la península antártica y las islas cercanas. En la temporada 2012-2013, el 76,6\% de los desembarcos ocurrió en solo 200 hectáreas.

Otro factor que incrementa el nivel de riesgo de alteración del medio ambiente antártico es el desarrollo de la tecnología, que ha facilitado los viajes a la Antártica, permitiendo que cada vez más personas tengan acceso a ella. En consecuencia, si bien en principio los visitantes eran personas más bien interesadas en la ciencia y preocupadas por el medioambiente antártico (Bastmeijer, 2003: 92), hoy la posibilidad de visitar es accesible para el público general.

\section{Regulación de las actividades turísticas en el Sistema del Tratado Antártico}

El Sistema del Tratado Antártico (en adelante, STA) ha intentado de cierta manera anticipar las potenciales situaciones de perturbación del medioambiente antártico, acogiendo al turismo como una de las actividades permitidas en la Antártica. En específico, establece normas preventivas en el Protocolo sobre Protección al Medioambiente (1991, en vigor 1998, en adelante PM), artículos 3, 8 y 13. Éste dispone que la protección del medioambiente antártico y los ecosistemas dependientes y asociados debe ser una consideración fundamental para la planificación y realización de todas las actividades en el área del Tratado Antártico. Para ello las actividades deben planificarse y realizarse en base a información suficiente, de manera que se limite el

Número de página no utilizable para citar 
impacto perjudicial en el medioambiente y sistemas asociados. Existe una obligación de los Estados de asegurar que los procedimientos de evaluación del impacto en el medioambiente se apliquen a los procesos de planificación de sus actividades. Esto debe ser complementado con el artículo 13, el cual dispone que, para asegurar el cumplimiento del PM, cada parte tomará medidas adecuadas dentro del ámbito de su competencia, lo que incluye la adopción de leyes y reglamentos, actos administrativos y medidas coercitivas.

Los procedimientos mencionados en el artículo 8 se detallan en el Anexo I al PM, "Evaluación del Impacto sobre el Medio Ambiente". Este contempla en primer lugar una fase de evaluación preliminar para determinar si una actividad tendrá más que un impacto mínimo o transitorio. Para ello se realiza una descripción de la actividad y la consideración de alternativas e impactos que pueda tener. Si la actividad en efecto tiene menos que un impacto mínimo o transitorio, se puede realizar. Si tiene un impacto mínimo o transitorio procede una evaluación ambiental inicial, conforme al artículo 2.2, y la actividad podrá realizarse siempre que se establezcan procedimientos apropiados, que pueden incluir la observación, para evaluar y verificar el impacto de la actividad. Finalmente, si la actividad tiene más que un impacto mínimo o transitorio se realiza una Evaluación Medioambiental Global. Este proyecto es enviado a todas las partes y puesto a disposición público, recibiéndose comentarios durante 90 días. Luego se debe poner a disposición del Comité para la Protección del Medio Ambiente y las Partes al menos 120 días antes de la próxima reunión consultiva. Se evalúa la actividad en las reuniones consultivas.

Hasta el momento, se han enviado 382 Evaluaciones Ambientales Iniciales a las autoridades nacionales, concentrándose en cuatro países: Estados Unidos, Nueva Zelanda, Francia y Alemania (Ligget y Stewart, 2017: 496).

Por otro lado, a nivel de las Reuniones Consultivas del Tratado Antártico, si bien el turismo ha sido tratado en numerosas ocasiones, hasta el momento no se han dictado resoluciones vinculantes, sino que más bien recomendaciones y directrices.

En complemento al STA, el Código Internacional para los buques que operan en aguas polares (2014, en vigor 2017), adoptado por la Organización Marítima Internacional en el marco de la Convención de Derecho del Mar, contiene algunas medidas obligatorias para prevenir la contaminación ${ }^{2}$.

Si bien la regulación previa establece un marco legal, este es bastante amplio, ya que no hay consenso sobre qué elementos deben tenerse en consideración y ser analizados para determinar

2 Para una ilustración sobre estas medidas, visitar http://www.imo.org/es/MediaCentre/HotTopics/polar/Documents/How\%20the\%20Polar\%20Code\%20pro tects $\% 20$ the $\% 20$ environment $\% 20$ (Spanish\%20infographic).pdf

Número de página no utilizable para citar 
Regulación Chilena para las actividades turísticas en la Antártica Carolina Flores Barros

el nivel de impacto ambiental que presenta la actividad, y requiere de su implementación en cada uno de los países para que pueda ser operativa en la práctica. Además, no contempla los casos de operadores que realizan sus actividades bajo banderas de Estados que no son parte del STA. En vista de lo anterior, ha resultado clave la autorregulación que se ha dado la International Association of Antarctica Tour Operators (IAATO). Creada en 1991 por operadores estadounidenses para coordinar sus actividades, hoy reúne a 115 operadores en Antártica, promoviendo el concepto de desarrollo sostenible del turismo, orientado hacia el cuidado del medioambiente. Las compañías asociadas a IAATO representan el 100\% de las compañías comerciales de cruceros que circulan en la Antártica desde la temporada 2008/2009 y el 90\% de los visitantes de esta región (Bender, Crosbie y Heather, 2016: 2).

IAATO impone códigos de conducta, publicando documentos como la Guía para quienes organizan y conducen turismo y actividades no gubernamentales en la Antártica y también Directrices generales para visitantes a la Antártica. Estas normas son llevadas a la práctica a través de la supervisión de sus miembros, la realización de actividades turísticas guiadas y la provisión de información a los turistas antes de su llegada al continente. IAATO ha contribuido al STA participando activamente en las Reuniones Consultivas sobre el Tratado Antártico, aportando información sobre turismo.

\section{Caracterización del turismo vinculado a Chile en la Antártica}

Chile cuenta con ventajas comparativas para el desarrollo de las actividades turísticas antárticas. Su cercanía geográfica con el continente, además de la existencia del Aeródromo Teniente Rodolfo Marsh, en la base "Presidente Eduardo Frei Montalva", lo constituyen como uno de los principales países facilitadores de actividades turísticas. A modo ilustrativo, en el año 2015, Aerolíneas DAP realizó 64 operaciones aéreas, en las cuales se transportaron 5.191 pasajeros (Dirección Antártica, Ministerio de Relaciones Exteriores, 2017: 63). Incluso, en el año 1982, se estableció una casa de huéspedes para acomodar turistas en dicha estación (Ligget, McIntosh, Thompson et al, 2011: 358) ${ }^{3}$. A lo anterior se suma la capitanía de puerto "Bahía Paraíso", de la Armada de Chile, ubicada en la base "Presidente Gabriel González Videla". Esta se ha constituido como uno de los cinco destinos más visitados de la Antártica, desembarcando alrededor de 6.000 turistas en cada verano austral (Dirección Antártica, Ministerio de Relaciones

3 Esta no continuó con sus servicios turísticos cuando la Fuerza Aérea Chilena se negó a transportar turistas en sus vuelos oficiales.

Número de página no utilizable para citar 
Exteriores, 2017: 65) ${ }^{4}$. Además, existen tres operadores chilenos afiliados a IAATO. Estos son DAP Antartica, Antarctica21 y SIM Expeditions. Las dos primeras ofrecen turismo antártico a través de la vía aérea desde la ciudad de Punta Arenas, mientras que la tercera realiza sus viajes por vía marítima, en yates, desde la ciudad de Puerto Williams. Además, existe un operador estadounidense Antarctic Logistics \& Expeditions (ALE), que opera en forma permanente desde Punta Arenas, ingresando turistas a través de la vía aérea, realizando actividades en la base chilena Glaciar Unión.

El turismo que opera desde Chile continental hacia el Territorio Antártico es principalmente Aéreo, y la cantidad de embarcaciones que se dirigen a la Antártica desde Punta Arenas es pequeña en comparación a la que opera desde Ushuaia, Argentina (utilizada como puerto de acceso a la Antártica por el 90\% de los cruceros antárticos) (Vereda, 2008: 200). La concesionaria portuaria de Punta Arenas, EPAUSTRAL informa que sólo hay una empresa, de origen holandés, que viaja a la Antártica con fines turísticos desde Punta Arenas a través de la vía marítima, la cual no pudo operar en la temporada 2018-2019, por problemas técnicos ${ }^{5}$. Sin embargo, la participación de Chile en el turismo antártico no sólo se restringe a la salida de embarcaciones desde puertos nacionales, ya que dada la cercanía geográfica del país con el continente, y su reclamación de soberanía en Antártica Chile ha intervenido activamente en el rescate de embarcaciones accidentadas ${ }^{6}$ y además se constituye como un país receptor de turistas en las bases que se encuentran en el territorio que reclama y de apoyo ante potenciales emergencias que pudieran observarse durante el desarrollo de actividades turísticas.

Respecto a la consagración legal de la prevención del medioambiente antártico y específicamente la implementación de las normas contenidas en el PM y su Anexo I, Chile ha sostenido como uno de sus objetivos la protección global del medioambiente antártico y los ecosistemas dependientes y asociados en el marco del Protocolo del Tratado Antártico sobre protección del medioambiente. En esta línea, ha buscado dar cumplimiento a las normas sobre prevención del daño ambiental observables en el Sistema del Tratado Antártico. Desde el establecimiento del Protocolo al Tratado Antártico sobre Protección del Medio Ambiente (1991, en vigor 1998), la evaluación ambiental preventiva de las actividades a realizarse en la Antártica se realizó a través del Comité Nacional para la Evaluación del Impacto Ambiental sobre el Medio Ambiente Antártico (CONAEIA), dependiente de la Comisión Nacional del Medioambiente. En el año 2010, con la entrada en vigor de la ley No 20.417, que Crea el Ministerio, el Servicio de Evaluación Ambiental y la Superintendencia del Medio Ambiente, el Ministerio del Medio Ambiente creó el

4 En la temporada 2015-2016 arribaron a la base Gabriel González Videla un total de 3.286 turistas, de los cuales 3.284 eran extranjeros.

5 Información obtenida a través de comunicación por correo electrónico.

6 La actividad de salvataje se remite a fines del siglo XIX. En 1896 se creó para estos fines la Compañía de Salvatajes de Punta Arenas. Entre 1896 y 1923 se rescataron 36 vapores (Instituto Antártico Chileno, 2013). 
Comité Operativo para la Evaluación del Impacto Ambiental sobre el Medio Ambiente Antártico (COEIA), a través de Resolución Exenta N¹.396. Ésta se limita a crear el Comité, definiendo las autoridades que forman parte de él, para la evaluación del impacto ambiental sobre el medio ambiente antártico, sin especificar sus funciones. Dentro de su composición se encuentra un representante de la Oficina Nacional de Turismo. El Comité no tiene un cronograma fijo de funciones, reuniéndose una o dos veces al año.

El Comité certifica el cumplimiento de las normas internacionales de todas las actividades que se realizan en la Antártica, conforme a lo indicado en el PM. Evalúa iniciativas chilenas y aquellas que se realizan en el Territorio Chileno Antártico. Esta evaluación no conlleva un acto administrativo de aprobación o reprobación de la actividad. En consecuencia, quienes deseen realizar actividades o proyectos en el Territorio Antártico Chileno deben rellenar un formulario web, donde se detalla el impacto ambiental de éstos. El COEIA trabaja en base a un documento elaborado por una corporación el año 2008, donde se especifican los elementos a considerar para determinar qué impacto tendrá cada actividad. Lo anterior se realiza en base a una tabla de valoración, que mide las variables reversibilidad, acumulación, extensión espacial, intensidad, duración y retardo.

Desde el año 2011 se han evaluado 44 actividades o proyectos y cinco se encuentran actualmente en evaluación. Éstos son clasificados según sus características en deportivos, de infraestructura, topo-batimetría, turísticos, culturales, científicos, logísticos, artísticos, levantamiento de información y estudio de suelos. De ellos, 13 son clasificados como turísticos. Sin embargo, de la información proporcionada sobre estos proyectos surgen algunas observaciones.

En primer lugar, llama la atención de que a pesar de lo dispuesto en los artículos 8.2 y 13 del PM y su Anexo I, Chile no haya optado por regular en forma estable los estudios de impacto ambiental en la Antártica, que haya optado por establecerlo a nivel reglamentario, en un acto administrativo que no ha pasado ni siquiera por el órgano contralor y que además es bastante escueto, no especificando las funciones del COEIA ni el procedimiento de evaluación. Esto ha sido desarrollado a través de documentos sin consagración legal. Cabría preguntarse las razones de ello, ya que en este caso la prevención de cualquier daño, como objetivo común de las partes de STA, no parece tener reparos como otro tipo de actividades, que pueden entrar en potencial conflicto con el artículo IV del Tratado Antártico. Es más, el PM expresamente ordena su regulación. En la práctica países como, Australia, Alemania, Países Bajos, Nueva Zelanda, Noruega, Suecia y el Reino Unido ya han contemplado en sus sistemas jurídicos la regulación de los estudios de Impacto Ambiental (Ligget y Stuart, 2017: 496).

Número de página no utilizable para citar 
Esto ha dado lugar a que no sea claro qué tipo de proyectos o actividades son aquellos que deben ser sometidos a Evaluación Ambiental Inicial, ni si este sometimiento es obligatorio. El COEIA cuenta con un sitio web que aún se encuentra en desarrollo, por lo que la información sólo está disponible al público mediante consulta directa. A lo anterior se suma una ambigüedad en los criterios de clasificación de las actividades. A modo de ejemplo, hay actividades que son clasificadas como culturales, mientras que otras se catalogan como artísticas. Además, algunas actividades que son clasificadas como "deportivas", también podrían ser consideradas como turísticas. Si definimos las actividades turísticas como aquellas actividades que son organizadas (comprendiendo gubernamentales, no gubernamentales o comerciales) en la región Antártica, entendiéndose como tal al sur de los 60 grados de latitud sur, con el propósito de disfrutar (comprendiendo placer, aventura o educación) valores Antárticos específicos (áreas silvestres, fauna, lejanía, situaciones extremas) en cualquiera de sus formas (Machiel Lamers, 2009:13), entonces actividades como competencias pedestres o circuitos deportivos deberían ser clasificados como actividades turísticas y no como actividades deportivas.

Sin perjuicio de las debilidades observadas en la consagración jurídica de la prevención del daño ambiental en Territorio Chileno Antártico, existen algunas iniciativas políticas que han buscado potenciar esta actividad bajo ciertos principios de prevención. Así, en el "Plan Estratégico Antártico 2015-2019”, elaborado por el Consejo de Política Antártica ${ }^{7}$, se establece como objetivo el fortalecimiento de la Región de Magallanes y Antártica Chilena como puerta de entrada a la Antártica. Se reconoce como un enclave operativo y logístico de las expediciones antárticas que tengan como destino la Isla Rey Jorge, la Península Antártica y el resto del continente. Dentro de este objetivo se contempla "Efectuar estudios destinados a establecer bases actualizadas para una Política Nacional de Turismo Antártico controlado.” En el marco de lo anterior, el 5 de abril de 2016 la Dirección Antártica del Ministerio de Relaciones Exteriores organizó un taller sobre turismo antártico, cuyos resultados fueron expuestos en la XXXIV RCTA realizada en Santiago en 2016.

El Consejo de Política Antártica también aprobó el documento "Chile en la Antártica. Visión Estratégica al 2035”, donde se identifica dentro de las proyecciones al futuro, la importancia de construir infraestructura para el apoyo logístico al turismo, además de aumentar la infraestructura hotelera en Punta Arenas, buscando consolidar a Punta Arenas como puerta de entrada a la Antártica. En el marco de la participación de Chile en el STA se busca potenciar las discusiones sobre turismo, que involucre el concepto de turismo controlado.

\footnotetext{
Es el órgano encargado de elevar al conocimiento y decisión del Presidente de la República sus recomendaciones para el desarrollo de la Política Antártica Nacional. Se reúne para adoptar o conocer las materias de mayor relevancia política y trascendencia internacional.
} 
Por su parte, la Política Antártica Nacional de 2017 reconoció dentro de sus objetivos la protección y promoción del cuidado del medioambiente antártico, remarcando que para ello es importante la existencia de un procedimiento claro para la aprobación de las actividades en la Antártica, en el marco de los Estudios de Impacto Ambiental. Específicamente, uno de sus objetivos es "facilitar el desarrollo de actividades económicas permitidas por el Sistema del Tratado Antártico, incluyendo la pesca sostenible y responsable, así como el turismo controlado y sustentable". Respecto a ello, menciona "En el ámbito del turismo antártico, Chile está consolidándose como un proveedor de servicios turísticos, en particular a través de la modalidad de Aero crucero. Es fundamental que esta industria sea controlada y sustentable, para minimizar su impacto sobre el medioambiente antártico. Para ello, es indispensable contar con una política nacional en materia de turismo antártico, que establezca parámetros dentro de los cuales se desarrollarán estas actividades."

En la práctica esto se ha ido potenciando a través de programas regionales tales como el Programa Estratégico Regional "PER Magallanes Experiencia Antártica", que tiene como propósito posicionar a la Región como referente mundial del turismo de intereses especiales, para aumentar el valor agregado de los visitantes internacionales en la economía de Magallanes, y también la promoción de Puerto Williams como la ciudad más austral del mundo.

\section{Conclusiones}

A nivel internacional se puede observar la existencia de un marco legal sobre prevención del potencial daño ambiental proveniente de actividades turísticas. Este se conforma principalmente por el PM y su Anexo I, que establecen el sistema de Evaluación del Impacto sobre el Medio Ambiente. Además, se complementa por las normas del Código Internacional para los buques que operan en aguas polares y los códigos de conducta y directrices de IAATO.

Sin perjuicio de lo anterior, este marco teórico requiere de implementación en los Estados para ser operativo, y demanda detallar ciertos aspectos técnicos. Además, no se resuelve la situación de aquellos Estados que no son parte del STA.

Chile, por su proximidad geográfica e infraestructura, se configura como un actor clave en materia de turismo. Como puerta de salida del turismo, el país más bien ha optado por el tránsito

8 Esto mediante la reformulación de la definición de ciudad que entrega el Instituto Nacional de Estadísticas, regido por la Ley Na17.734. Bajo los parámetros de la Convención Europea de Ciudades de Praga de 1976, se define como ciudad aquellas entidades urbanas que posean entre dos mil y diez mil habitantes.

Número de página no utilizable para citar 
aéreo. Como puerta de entrada a la Antártica, recibe una gran afluencia de turistas por la vía aérea y marítima. A lo anterior se suma el rol del país en las operaciones de rescate en accidentes relacionados con esta actividad.

A nivel político se observa un reconocimiento y compromiso por parte de Chile en cuanto a la implementación de la normativa internacional relativa a prevención del daño ambiental proveniente de actividades turísticas, tanto a nivel central, a través de la Política Antártica Nacional y el Plan Estratégico Antártico 2015-2019, como a nivel regional por medio del PER Magallanes Experiencia Antártica. Sin embargo, para estar en línea con la política de desarrollo sostenible y controlado del turismo, las políticas aún deben desarrollarse.

Se plantea como necesaria la promulgación de normativa con mayor estabilidad y permanencia, a través de una ley que regule en forma clara las funciones y atribuciones de la entidad anterior, incluyendo la facultad de rechazar actividades turísticas que no se ajusten a los parámetros ambientales necesarios para operar en la Antártica. También se requiere un trabajo institucional para dar publicidad a las evaluaciones del COEIA, con criterios y estadísticas precisas y a disposición del público general. También resultaría relevante considerar todos los flancos de la actividad turística, y no solo la circulación a través del territorio chileno continental. Solo incorporando las observaciones anteriores, se podría dar cumplimiento a lo dispuesto en los artículos 8.2 y 13 del PM en forma satisfactoria.

\section{Referencias bibliográficas}

BAstMeijer, Kees y Ricardo Roura (2004). "Regulating Antarctic Tourism and the Precautionary Principle", The American Journal of International Law, 98: 763.

BASTMEIJER, Kees (2003). "Tourism in Antarctica: Increasing Diversity and the Legal Criteria for Authorisation", New Zealand Journal of Environmental Law, 7: 92.

BENDER, Nicole; Kim Crosbie y Heather Lynch (2016). "Patterns of Tourism in the Antarctic Peninsula Region: a 20-Year Analysis.” Antarctic Science, 28 (3): 194-203. doi:10.1017/S0954102016000031.

CORFO (Corporación de Fomento a la Producción, Chile) (2018). Resumen Programa Estratégico de Turismo "Magallanes Experiencia Antártica" para Convocatoria IPRO 2018.

CORPORACIÓN CHILEAMBIENTE (2008). "Criterios para la evaluación ambiental de las actividades a realizar en Antártica", Informe Final. 
Regulación Chilena para las actividades turísticas en la Antártica Carolina Flores Barros

DiRECCIÓn DE ANTÁRTICA, Ministerio de Relaciones Exteriores (2017). "Memoria Antártica Nacional. Campaña Antártica 2015-2016”. https://minrel.gob.cl/minrel/site/artic/20121010/asocfile/20121010172919/memoria_ant_rtica_201 5_2016.pdf

FEDCHUK, Andrii (2013). "The Development of Tourism at Faraday/Vernadsky Station: Changes in Governance and Visitor Patterns.” Polar Record, 49 (3): 286-90. DOI:10.1017/S0032247412000599.

HEMMINGS, Alan y Ricardo Roura (2003). "A square peg in a round hole: fitting impact assessment under the Antarctic Environmental Protocol to Antarctic tourism", Impact Assessment and Project Appraisal, 21: 13-24. DOI: $10.3152 / 147154603781766455$

HuGHES, Kevin et al. (2018). "Antarctic environmental protection: Strengthening the links between science and governance", Environmental Science \& Policy, 83: 86-95. doi: https://doi.org/10.1016/j.envsci.2018.02.006.

INSTITUTO ANTÁRTICO CHILENO (2013). “Guía circuito antártico”. http://www.inach.cl/inach/wpcontent/uploads/2013/04/guia-circuito.pdf

LAMERS, Machiel (2009). "The future of Tourism in Antarctica. Challenges for Sustainability". PhD thesis, Maastricht University.

LAMERS, Machiel, y Bas Amelung (2011). "Sustainable Tourism Development in Antarctica". Polar Tourism: A Tool for Regional Development, editado por Dieter Mueller y Alain Grenier, 207-222. Montreal: Presses de l'Universite du Quebec.

LIGGETT Daniela (2011). "Tourism in the Antarctic: Modi Operandi and Regulatory Effectiveness", Tourism Management, 32: 357-366.

LIGGETT, Daniela et al. (2011). "From frozen continent to tourism hotspot? Five decades of Antarctic tourism development and management, and a glimpse into the future". Tourism Management, 32: 357-366.

LIGGETT, Daniela y Emma Stewart (2017). "Sailing in Icy Waters: Antarctic Cruise Tourism Development, Regulation and Management". Cruise Ship Tourism, editado por Ross Dowling y Clare Weeden, 484-504. Reino Unido: CABI.

Retamales EsPinOZA, José (2009). "Política Antártica Nacional: Mecanismos de Aplicación". Revista Diplomacia, 121: 9-26.

Servicio Nacional de Turismo (2014). Plan de acción Región de Magallanes y de la Antártica Chilena. Sector Turismo 2014-2018. Chile.

TURner, John et al. (2014). "Antarctic Climate Change and the Environment: An Update", Polar Record 50 (3): 237-59. doi:10.1017/S0032247413000296.

VEREDA, Marisol (2008). "Tierra del Fuego y Antártida. Un inventario de recursos turísticos desde la idea de la complementariedad", Estudios y perspectivas en turismo, 17 (3): 199-225.

Número de página no utilizable para citar 